\title{
Le genre dans les manuels scolaires français. Des représentations stéréotypées et discriminatoires
}

\section{Sabrina Sinigalia-Amadio}

\section{(2) OpenEdition \\ Journals}

Édition électronique

URL : http://journals.openedition.org/trema/2665

DOI : 10.4000/trema.2665

ISSN : 2107-0997

\section{Éditeur}

Faculté d'Éducation de l'université de Montpellier

Édition imprimée

Date de publication : 1 décembre 2011

Pagination : $98-115$

ISSN : 1167-315X

Référence électronique

Sabrina Sinigalia-Amadio, « Le genre dans les manuels scolaires français.

Des représentations stéréotypées et discriminatoires », Tréma [En ligne], 35 - 36 | 2011, mis en ligne le

01 décembre 2013, consulté le 10 décembre 2020. URL : http://journals.openedition.org/trema/2665 ; DOI : https://doi.org/10.4000/trema.2665

Ce document a été généré automatiquement le 10 décembre 2020.

Trema 
Le genre dans les manuels scolaires français. Des représentations stéréotypées et discriminatoires

Sabrina Sinigalia-Amadio

I. Introduction 
1 L'étude ${ }^{1}$ sur laquelle repose cet article a été réalisée en 2008 pour la Haute Autorité de Lutte contre les Discriminations et pour l'Égalité (HALDE $\left.{ }^{2}\right)$ L'équipe pluridisciplinaire engagée dans l'enquête et dirigée par Pascal TISSERANT et AnneLorraine WAGNER se composait de juristes, psychologues, cognitivistes et sociologues. La commande de la HALDE était double: d'une part, évaluer le traitement de la discrimination dans les manuels scolaires d'éducation civique censés initier et sensibiliser les élèves aux questions sociales et citoyennes dont fait partie la lutte contre les discriminations et d'autre part, repérer les éventuels stéréotypes véhiculés à l'encontre de différents " groupes» sociaux au sein de manuels de

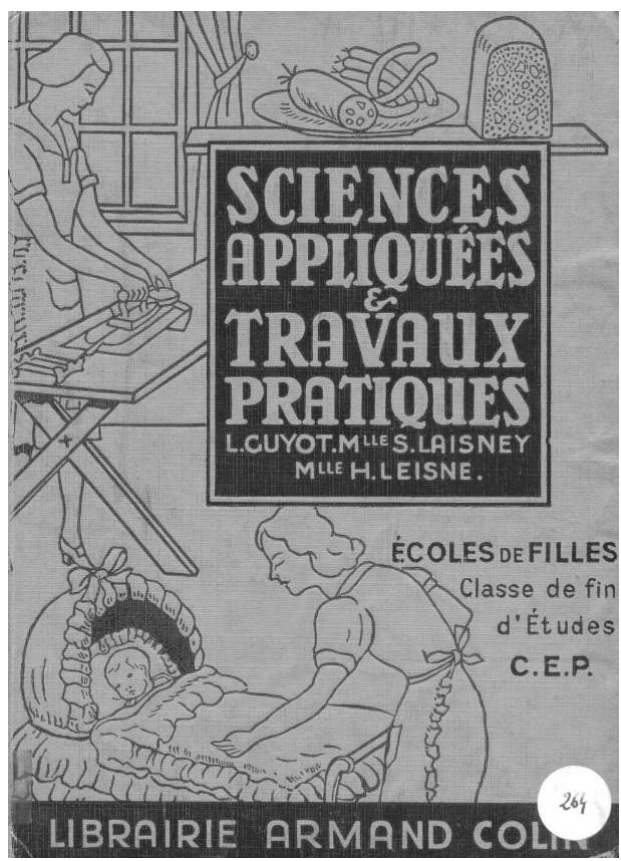
différentes disciplines. Quelle place y est donnée aux femmes et aux hommes ? Qu'en est-il de ce que l'on appelle la « diversité » dite ethnique ou culturelle? Quelle vision des différents âges et notamment des seniors »? Quelles représentations des différentes sexualités (hétéro-, homo-, bi-sexuelle) ? Enfin quelle visibilité des différents handicaps?

2 Sur le plan méthodologique, nous avons procédé à l'analyse de 29 manuels de diverses disciplines (français, mathématiques, sciences de la vie et de la terre, anglais, histoire/ géographie, éducation civique, sciences économiques et sociales) enseignées au collège et au lycée, dans l'enseignement général et professionnel. Les éditions retenues figurent parmi les plus utilisées dans les établissements scolaires (Cf. corpus en bibliographie). Chacune des disciplines indiquées a été choisie pour une raison précise. L'éducation civique (juridique et sociale) et les sciences économiques et sociales ont au programme la question de la discrimination; le français et l'histoire/géographie ont fait l'objet de préconisations dans divers rapports et études réalisés depuis les années 1970, et tout particulièrement depuis celui e Simone RIGNAULT et Philippe RICHERT (1997) ; l'anglais, la langue vivante la plus enseignée en France, aborde de nombreuses problématiques de l'étude; enfin les manuels de sciences de la vie et de la terre et de mathématiques permettent d'interroger la supposée neutralité de ces disciplines: décortiquer les exercices dans lesquels apparaît une mise en contexte permet en effet de déconstruire cette représentation erronée des sciences dites exactes. Les manuels ont été analysés qualitativement (étude des situations et contextes de mise en scène des individus) et quantitativement (comptage d'occurrences, de photos, de prénoms/noms). Afin de compléter notre analyse, et notamment parce que le manuel ne constitue pas à lui seul l'enseignement transmis dans les établissements et les classes, nous avons également soumis un questionnaire accessible en ligne aux enseignant-e-s $(\mathrm{N}=190)$ et réalisé des entretiens avec 18 enseignant-e-s, 34 élèves et 8 éditeurs-éditrices afin de solliciter leur point de vue. Cela dit, l'essentiel de l'analyse est ici centrée sur les manuels (contenu et forme). 
3 Aucune étude à ce jour ne considérait l'ensemble de ces critères (origine culturelle réelle ou supposée, handicap, vieillesse, genre, sexualité), les études sur les stéréotypes dans les manuels concernant essentiellement l'image des femmes. Ainsi, en 1979 paraît l'ouvrage d'Annie DeCrouX-MASson, Papa lit, maman coud. Les manuels scolaires en bleu et rose. Depuis Simone RIGNAULt et Philippe RICHERT, entre autres, ont rappelé la persistance de cette inégalité de traitement dans un rapport intitulé La représentation des hommes et des femmes dans les livres scolaires (1997). Plus récemment, Amandine BERTON-SCHMITT s'est intéressée à La place des femmes dans les manuels d'histoire du secondaire (2005). Par ailleurs, des travaux ont permis d'avoir une vision plus étendue des stéréotypes de genre véhiculés par la littérature destinée aux enfants (CROMER, Turin, 1997, 1998; BRUGEILLES, CROMER, 2005 ; Détrez, 2006). Sans chercher à mesurer strictement les écarts depuis les premiers travaux et les suivants (l'absence de méthodologie commune rendant la comparaison terme à terme impossible), il s'est agi de considérer l'existant et, lorsque cela était possible, de comparer les analyses.

Ces considérations méthodologiques et théoriques posées, il convient de revenir dans un premier temps sur les grands résultats de l'enquête avant d'entrer de manière plus fine dans l'analyse des stéréotypes genrés.

\section{Quelques grands résultats de l'enquête}

Concernant le premier objectif de l'enquête («identifier le traitement de la question de l'égalité et des discriminations dans les manuels scolaires d'éducation civique", principalement en $5 \mathrm{e}$, qui consacre un tiers de son programme à ces questions), nous avons pu observer une relative discrétion au sujet de la politique de lutte contre les discriminations, nationale comme internationale (convention de l'ONU, l'UNESCO, commissions européennes), pourtant présentée comme une priorité. Sur l'ensemble des manuels, les politiques de lutte contre les discriminations ne couvrent que quelques rares pages, ce qui en limite la portée. Celles-ci ne font souvent que rappeler la prohibition de la discrimination sans réellement proposer des modalités de comportements ou de représentations non discriminatoires, en dépit des objectifs affichés dans les programmes ( Il convient aussi de [...] faire réfléchir [les élèves] sur les politiques et les actions menées pour lutter contre les discriminations et le racisme " ${ }^{3}$ ). Au point que certain-e-s enseignant-e-s disent se tourner vers d'autres supports pour travailler ces questions, autres livres, textes, voire des supports audiovisuels (spots publicitaires, extraits de films, etc.). Cela suppose une sensibilisation et une formation préalables à la question.

Concernant le second objectif ( repérer les représentations et stéréotypes dans les manuels scolaires référant aux critères de discrimination qui relèvent du domaine de compétence de la HALDE »), les résultats sont moins univoques. Au premier abord, les enseignant-e-s rencontré-e-s qui envisagent le manuel scolaire comme agent de transformation sociale, disent globalement s'en satisfaire, même si ponctuellement ils/ elles recourent à des supports complémentaires, comme indiqué précédemment. Cependant, les manuels étudiés n'offrent qu'une représentation stéréotypée des individus : qu'il s'agisse des femmes, des seniors, des personnes en situation de handicap, des «minorités visibles ${ }^{4}$ et plus encore des personnes homosexuelles, elles sont globalement sous-représentées et lorsqu'elles le sont, les stéréotypes opèrent une très nette réduction identitaire des personnes. 
6 Concernant les femmes, elles sont toujours nettement moins représentées que les hommes et n'évoluent pas dans toutes les sphères sociales: quasi absentes du champ politique et intellectuel, elles sont surreprésentées au sein de l'univers domestique et l'emploi des femmes se confine dans des domaines traditionnellement réservés aux femmes.

7 Les «minorités visibles » connaissent un traitement différent selon les disciplines: les manuels de langue ont davantage tendance à les représenter que les autres, tout particulièrement ceux d'histoire/géographie. Les manuels de français ne laissent pratiquement aucune place aux auteur-e-s, illustrations ou textes étrangers francophones ou autres (traduits). En mathématiques ou Sciences de la Vie et de la Terre, il est plus difficile de lire cette représentation. Cependant l'utilisation des prénoms dans les exercices de mathématiques est tout à fait édifiante puisque les prénoms à consonance étrangère sont extrêmement rares (autour de $2 \%$ ), et lorsqu'ils sont présents, la mise en scène témoigne régulièrement d'un cliché exotique (Ali Baba et les quarante voleurs, un cheikh doit partager ses dix-sept chameaux entre ses trois fils). Enfin, les minorités évoquées ne concernent que quelques parties du monde (l'Afrique de Nord, le Moyen Orient), quelques «types » de personnes (les Noir-e-s d'Afrique Noire, des DOM TOM ou d'Amérique) et une religion (l'islam).

8 Le handicap et les personnes handicapées sont quasiment absentes des manuels scolaires. Certes, les illustrations ne mettent pas uniquement en scène des personnes en difficulté et dépendantes de personnes valides et des exemples positifs pointent les compétences des personnes handicapées et la réussite exemplaire de quelques-un-e-s. Cependant, leur apparition sert toujours strictement à interroger la question du handicap, opérant par là même une réduction identitaire forte à l'encontre des individus (leur handicap occupant le devant de la scène). Enfin, il importe de relever que seuls les handicaps les plus visibles socialement sont évoqués (psychomoteurs et trisomie 21).

9 Absente des programmes 5 , la diversité des sexualités est également absente des manuels. La société présentée aux élèves est uniquement hétérosexuelle. Aucun couple homosexuel n'est figuré. Sur les 29 manuels et 3097 illustrations, on trouve seulement une photographie prise lors de la Gay Pride à Paris ouvrant un chapitre sur la diversification des objets et formes de l'action collective (Manuel de Sciences économiques et sociales de Terminale, Hatier, 2007). Les thématiques qui laissent apparaître l'existence des personnes homosexuelles sont toujours spécifiques : la gay pride, le PACS, l'épidémie de Sida, etc. Les manuels de Science de la vie et de la terre sont à cet égard significatifs puisque l'éducation à la sexualité et à la prévention se fait suivant un modèle hétéronormé : à l'adolescence, « les garçons recommencent à regarder plus attentivement les filles et inversement... » (Science de la vie et de la terre SVT 4e, Nathan, 2007). Il en va de même concernant la contraception et la procréation médicalement assistée, qui ne concernent que des couples hétérosexuels. Il appartient aux enseignant-e-s qui souhaitent avec leurs élèves travailler ces questions et d'autres (discrimination et égalité des droits, homophobie et agressions verbales et physiques...) de se saisir d'autres supports (films, ouvrages, expositions...).

10 Les senior-e-s, quant à eux/elles, sont peu représenté-e-s, rarement plus de deux fois par manuel, à travers des contextes stéréotypés. Ils apparaissent ainsi le plus souvent en contexte de grand-parentalité (observant quelques photos anciennes avec un enfant, par exemple) ou dans des situations dépréciatives, de dépendance, avec des problèmes de santé, d'isolement, de déficiences physiques. Présenté-e-s en activité, ils/elles évoquent 
d'autres époques (photos en noir et blanc à l'appui) ou sont dans une position prestigieuse, figures politiques connues (Jean JAURÈs, Kofi ANNAN, etc.) ou personnes célèbres (Agatha CHRISTIE à son bureau, Alexander-Graham BELL avec un téléphone) ou comme professionnels au statut important (juge, maire, ministre, etc.).

La vision du monde donnée aux élèves à travers les manuels scolaires renforce souvent les clichés, minimisant, voire omettant l'existence d'une partie de sa réalité : une activité professionnelle des femmes réduite et circonscrite, une faible diversité dite ethnique et culturelle, une absence de diversité d'orientations sexuelles, des personnes en situation de handicap quasi inexistantes, des senior-e-s peu représenté-e-s.

$12 \mathrm{Au}$ sein des stéréotypes et discriminations toujours d'actualité dans les manuels utilisés actuellement pour la formation des élèves, quels sont précisément ces stéréotypes liés au sexe qui émaillent les textes et illustrations des manuels?

\section{Stéréotypes et discriminations liés au sexe}

$13 \mathrm{Au}$ terme de l'étude réalisée, le premier constat est que les pratiques semblent se transformer plus rapidement que les représentations sociales, notamment celles véhiculées dans les manuels scolaires. En dépit de l'évolution de la place des femmes ces cinquante dernières années (FRAISSE, 1998; PERROT, 1998; DUBY, PERROT, 2002), plonger dans l'univers des manuels scolaires c'est opérer un bond dans le temps : le temps où les femmes s'occupaient essentiellement des enfants et des activités domestiques, et où les salariées étaient principalement infirmières, sage-femmes ou institutrices ${ }^{6}$. L'accès des femmes à la scolarisation et la réussite scolaire (BAUDELOT, ESTABLET, 1992 ; TERRAIL, 1997 ; DJIDER, MURAT, ROBIN, 2003 ; BAUDELOT, ESTABLET, 2007) ou à une plus grande diversification d'emplois' (BATTAGLIOLA, 2000; MARUANI, 2000) ne semblent pas infléchir cette représentation dominante.

Les recommandations formulées à destination des pouvoirs publics, précisément du ministère de l'Éducation nationale, et du monde de l'édition depuis les années 1970, ne semblent visiblement pas entendues et encore moins suivies. La présentation aux élèves du collège, et des adultes qui y travaillent, est sur ce point éclairante.

\section{1 Les manuels d'éducation civique : peut mieux faire...}

Le manuel Éducation civique $6 e$ : Grandir ensemble 2005, présente sur une double page « qui travaille au collège » afin que les élèves, fraîchement arrivés de l'école primaire puissent saisir toute la complexité et la diversité des adultes qui vont désormais les encadrer.

Le message transmis aux élèves en douze vignettes est que les fonctions d'ordre (Conseiller principal d'éducation), de gestion (gestionnaire) ou de direction (principal et principal adjoint d'un établissement, chef de cuisine, professeur principal) reviennent aux hommes, alors que les fonctions intermédiaires de soin, d'éducation, d'assistance ou de service (documentaliste, conseillère d'orientation psychologue, infirmière, serveuse de la cantine, assistante sociale) sont attribuées aux femmes. Les objets associés aux personnages renforcent encore le clivage sexué : stylo et ordinateur ornent le bureau du gestionnaire, tandis que l'assistante sociale téléphone à proximité d'une plante verte.

16 Le reste du manuel est dans la même veine, avec principalement des photographies d'hommes en situation de travail (un enseignant, un policier, deux chirurgiens, un 
policier, trois ouvriers sur un chantier, un éboueur, deux éboueurs, un luthier) et quelques rares femmes dans des professions qui leur sont traditionnellement associées (une enseignante, une secrétaire de mairie du bureau d'état civil, une agente de police faisant traverser des enfants à la sortie d'une école).

Cette double page et l'ensemble du manuel d'éducation civique sont d'autant plus difficiles à comprendre que le manuel pour les 3e (Éducation civique. Grandir ensemble, 2007), par les mêmes auteurs (auxquels s'ajoute un cinquième) présente quelques pages intéressantes, sur le droit de vote des femmes, la loi sur la parité, la place des femmes dans la société française. Par ailleurs, une présentation de quelques femmes célèbres a le souci de féminiser les fonctions: "Olympe de GOUGE (1748-1793) auteure d'une Déclaration des droits de la femme pendant la Révolution française » - même si on regrette que le titre de l'œuvre n'ait pas été respecté dans son intégralité -, « Simone de BEAUVOIR (1908-1986), écrivaine et féministe », « Françoise GIROUD (1916-2003), journaliste et ministre " et "Simone WEIL (née en 1927, Ministre et députée européenne »). Cependant, à nouveau certaines pages renforcent la sexualisation stéréotypée des professions, par exemple lorsqu'il s'agit de présenter, photos à l'appui, les fonctionnaires, avec "un préfet", " un enseignant", " un policier ", " un contrôleur des services sanitaires », « un pompier » et « une infirmière »!

On aurait pu penser à une utilisation du masculin, supposé neutre, mais le cas de l'infirmière infirme cette éventualité. Nous sommes simplement face à une vision stéréotypée classique des fonctions des unes et des autres. Les habitudes langagières ont bien du mal à être corrigées, même dans un manuel censé traiter spécifiquement de ces questions. Le manuel pour les 5e de cette même collection présente une page similaire, frôlant la caricature ${ }^{8}$.

\section{2 Les manuels de SVT : des chapitres un peu trop survolés...}

Les manuels de SVT ne sont pas beaucoup plus enclins à évoquer la diversité sexuée des professions. A titre d'exemple, dans le manuel de SVT 4e, Nathan, programme 2007, chaque chapitre se clôt par un encadré "un métier» / " une passion». Ces encadrés présentent essentiellement des hommes (un ingénieur géologue, un volcanologue, un chauffagiste, un soigneur animalier, un acousticien, un endocrinologue). Les rares femmes apparaissent soit au second plan, comme dans l'encadré « une passion: L'aide humanitaire ", soit exerçant un métier " dit féminin » tel celui d'une sage-femme, qui explique à une mère comment s'occuper de ses triplés, ou d'une infirmière scolaire. Par ailleurs, on relève la présence d'une gynécologue. Si la gynécologie est un métier valorisé socialement (médecine spécialisée ${ }^{10}$ ), elle s'apparente, de manière plutôt traditionnelle et stéréotypique, à un champ médical "réservé » aux femmes avec un ensemble de situations associées comme la grossesse, les enfants, etc.). Tout au long des chapitres de ce manuel, la sexualité, le développement du corps (enfant - adolescent - adulte), la question de la gestion de la contraception et de la grossesse et, par extension, des enfants, sont systématiquement associés aux seules femmes. Les hommes (amants, conjoints, pères) sont totalement absents, comme s'il revenait uniquement aux femmes de se préoccuper du contraceptif à utiliser, de la grossesse à surveiller, etc. Enfin, si le gynécologue est une femme (chapitre 7), le neurochirurgien, lui, est un homme (chapitre 10). La différenciation sexuée est ainsi traversée par la hiérarchie de prestige du corps médical. Le chapitre 6 semble tout à fait singulier dans ce paysage sexiste. Il évoque « un 
métier, celui de Pisciculteur(trice)». Le titre semble soucieux d'une présentation paritaire, avec l'ajout de «trice» entre parenthèses, mais la présentation et la photographie viennent contredire cette possibilité d'identification et de projection de soi pour les filles car, pour être "pisciculteur(trice)», il faut être "robuste » comme en témoignent les quatre étudiants à la musculature imposante, qui pêchent des daurades royales.

Les femmes n'apparaissent réellement que dans les chapitres 7 à 9 consacrés à « La transmission de la vie chez l'Homme ", les neuf autres chapitres du manuel ne semblent concerner que les hommes. Il importe de rappeler que ce manuel, à l'instar de ceux cités pour les autres disciplines, ne constitue en rien une exception. Il est à l'image des autres manuels de la même discipline.

\section{3 Les manuels de mathématiques : doivent soigner les détails de la copie...}

Les manuels de mathématiques ne sont pas en reste quant à la diffusion des stéréotypes, certains énoncés tendant à renforcer une division sexuée du travail, des activités et espaces sociaux. Dans le manuel de Mathématiques 5e, Collection Phare, programme 2006, HACHETTE ÉDUCATION, on lit par exemple: «Un chocolatier prépare des paquets avec des chocolats noirs et des chocolats blancs ", «Dans un cybercafé, Quentin est resté connecté 2,6 h sur Internet », «François collectionne les voitures miniatures... Joséphine possède une importante collection de poupées », "Serge a planté dans son potager 3 rangées de 5 salades ", " Valérie a acheté des gommettes autocollantes pour décorer une carte pour la fête des mères ", "Simon possède 210 timbres dans sa collection", "le professeur a réparti les élèves... Quentin a lu 3/5 d'un livre...Pour une randonnée, à VTT, Théo a rempli sa poche à eau de son sac ", "Lucie, Léa et Ninon se cotisent pour offrir un cadeau à leur grand-mère ", "Cécile note dans un cahier le montant des achats qu'elle réalise (une robe pour le réveillon...) », " Ariane achète un sweat-shirt coûtant $48 €$ », «Un agriculteur possède 52 hectares ", " Katia veut diminuer ses envois de SMS », "Camille mesure les dimensions, en mètre, de sa cuisine de forme rectangulaire ", « le président d'un club de rugby », « le mercredi après-midi, Safi fait de la danse moderne », etc.

On observe une ségrégation sexuée des sports (ici, rugby vs danse moderne). Par ailleurs, dès qu'il s'agit de métiers de l'agriculture, de l'artisanat ou de construction, les prénoms sont masculins et dans tous les cas, on parle au masculin (Serge, un chocolatier, un agriculteur). Par contre, lorsqu'il s'agit de mesurer les dimensions de «sa » cuisine, les auteurs choisissent un prénom féminin. De plus, non seulement ce sont les filles qui " achètent", mais leurs achats sont souvent stéréotypés (des vêtements, un forfait téléphonique, etc.). Enfin, les " passions » des unes et des autres sont là encore édifiantes $\mathrm{du}$ point de vue d'une sexualisation des pratiques (poupées et gommettes pour les filles, voitures, timbres, livres et Internet pour les garçons). Des illustrations anodines contribuent «mine de rien » à la construction du genre : les filles sont invitées à entrer très tôt dans un rôle social de femme d'intérieur alors que les garçons peuvent développer leur imagination et se projeter dans des univers différents (BAERLOCHER, 2006 ; BAUDELOT et ESTABLET, 2007).

Les exercices proposant un traitement égalitaire font figure d'exception : "Cassandre a acheté $1,950 \mathrm{~kg}$ de figues... Thierry a acheté $1,480 \mathrm{~kg}$ de navets... Marion a acheté $1,280 \mathrm{~kg}$ 
de cèpes... Mario a acheté $2,350 \mathrm{~kg}$ de girolles... Juliette a acheté $480 \mathrm{~g}$ de cerises 》, « Trois amis, Wilfried, Thérèse et Géraud ont ramassé des champignons ", "Vivian, Franck et Laura se sont présentés à l'élection de délégués de classe ", "Valérie part en vacances à Papeete... Romain part en vacances à Paris... », « les parents de Louis veulent savoir dans quel collège... ». Ces exercices portent cependant sur des dimensions relativement asexuées ou aux enjeux moindres : les aliments, les vacances. On soulignera cependant le souci de lier les deux sexes à une élection de délégués (qui peut être considérée comme un premier niveau de socialisation politique) ou au choix d'orientation scolaire fait par des parents (et non exclusivement par la mère).

Quant aux contre-stéréotypes, ce sont les grands absents, avec quatre petits énoncés seulement sur l'ensemble du manuel : «Julie court les $100 \mathrm{~m}$ en $12,5 \mathrm{~s}$... Jonathan a acheté $1,45 \mathrm{~m}$ de tissus... », « Pierrick veut acheter un vase », "Carine désire acheter un lecteurenregistreur DVD à disque dur ", « En se rendant à son travail, Pauline fait une halte chez son cousin Romain... ». Ainsi les filles sont associées à des performances sportives (et non à une simple promenade), au monde technologique (un lecteur-enregistreur DVD à disque dur) et au monde du travail et les garçons achètent des objets traditionnellement associés à l'univers féminin (tissu et vase).

Les photographies renforcent les stéréotypes en ne mettant quasiment jamais les femmes en scène. Concernant les personnalités, aucune mention n'est faite de l'existence de femmes mathématiciennes. Les associations telles que Femmes et sciences, Femmes et mathématiques et Femmes ingénieurs (2006) sont pourtant en mesure de citer une longue liste de femmes scientifiques aux travaux majeurs dans leurs disciplines, telles que Sofia KOVALEVSKAIA ${ }^{11}$, Gabrielle-Émilie LE TONNELIER DE BRETEUIL ${ }^{12}$, une dizaine de lauréates du prix Nobel ou d'autres lauréates de prix divers, de thèses remarquées, reprises, etc. : Marie CURIE, née Sklodowska, Irène JOLIOT-CURIE, Maria GOEPPERT-MAYER, Dorothy CROWFOOT HODGKIN, entre autres ${ }^{13}$.

\section{4 Les manuels d'histoire/géographie : doivent poursuivre leurs efforts...}

Concernant les manuels d'Histoire/Géographie, les principaux constats du rapport RIGNAULT et RICHERT (1997) valent toujours. Les femmes restent très largement absentes de l'histoire politique. Lorsqu'elles le sont, elles sont plutôt des suiveuses, voire des objets de discours plutôt que des actrices de l'histoire. Ou alors, ne spécifiant pas l'impact de leur action, le manuel n'invite pas les élèves à s'arrêter sur le sens politique de leur engagement. Les femmes représentées le sont toujours plutôt sous forme de la femmeallégorie, icône ou emblème (la liberté, la république, la civilisation, des déesses grecques, romaines...), la mère de famille avec enfant, la «fille de ", «femme de » ou « mère de ", femmes-objet et/ou séductrices (valorisées par et/ou pour leur corps). Au mieux, on cite les sempiternelles femmes célèbres (Coco CHANEL, Marie cuRIE, Rosa LUXEMBOURG), Rares sont les figures féminines incluses dans l'Histoire (par exemple, dans Histoire/Géographie 4e, Hatier, collection Martin Ivernel, 2006, seule Louise Michel apparait comme telle, p.200 dans un chapitre sur la Commune de 1871); elles se trouvent généralement dans des chapitres « réservés » tels « les femmes dans la résistance » ou concernant «l'évolution des droits des femmes ». masculin présenté abusivement comme «universel»), les omissions de personnages 
importants sont toujours regrettables, comme Téroigne DE MÉRICOURT ou Olympes DE GOUGES ou encore les militants pour le droit des femmes tels CONDORCET ou le socialiste Charles FOURIER, ne citant dans le meilleur des cas que les traditionnelles George SAND et Flora TRISTAN.

\section{5 Les manuels de français : des connaissances à approfondir...}

Les manuels de français n'offrent qu'une place mineure aux femmes auteures et/ou à leurs œuvres. Français. Textes littéraires (livre unique, 2de, Hatier, 2006) est tout à fait représentatif. Sur les 356 documents répertoriés dans ce manuel de 574 pages, 35 concernent des femmes auteures et/ou leurs œuvres (soit moins de $10 \%$ ), dont une majorité apparaissent dans des séquences spécialement réservées à la question des femmes (chapitre 7 , séquence 21 "L'éducation des femmes, un débat moderne ${ }^{14}$ et séquence 22 «La place de la femme : écrits féministes du XVIIIe au XXIe siècle $»^{15}$ ). Sur ces 35, nous comptons des auteures récurrentes, Simone DE BEAUVOIR, Marguerite DURAS, COLETTE, Élisabeth BADINTER ou encore Gisèle HALIMI. Rares sont les documents abordant la situation dominée des femmes: une affiche du collectif féministe Guerrilla Girls titrée «Les femmes doivent-elles être nues pour entrer au Met. Museum? Moins de $3 \%$ des artistes des salles consacrées à l'art moderne sont des femmes, mais $83 \%$ des nus sont des nus féminins » et un extrait $D u$ côté des petites filles de Elena GIANINI BELOTTI.

Sur les 16 sujets d'exposés et/ou d'exploitation de documents proposés aux élèves, seuls deux portent sur une artiste ou un sujet concernant les femmes: «la vie et l'œuvre de Colette » et « le portrait féminin au XIXe siècle ». Enfin, sur les 87 notices biographiques recensées à la fin de l'ouvrage, seules 12 concernent des femmes (Laure ADLER, Mariama BÂ, Elisabeth BADINTER, Simone DE BEAUVOIR, COLETTE, Marguerite DURAS, Olympe DE GOUGES, Gisèle HALIMI, Sarah KANE, Mme DE LA FAYETTE, Virginia wOoLf et Marguerite YouRCENAR). Il est intéressant de remarquer que la notice de Simone DE BEAUVoIR précise qu'elle fut la compagne de Jean-Paul Sartre, sans réciproque dans celle de Jean-Paul Sartre. Il semble donc bien difficile, pour certains auteurs de manuels ou éditeurs, de se départir de certaines habitudes (toujours « femme de » sans jamais être « homme de »).

\section{Conclusion}

$29 \mathrm{Au}$ terme de cette analyse, nous pouvons d'abord observer qu'aucun «contrestéréotype » de type « papa coud et maman lit » n'existe à ce jour. Non qu'une inversion des rôles soit la solution aux inégalités de traitement des femmes et des hommes dans les manuels, même si ce type d'exemple permettrait déjà de présenter la diversité des modes de vie « négociés » (DE SINGLY, 1996) existants. Ensuite les représentations féminines et les modèles d'identification en direction des filles sont non seulement trop rares mais fortement stéréotypés. Les auteur-e-s des manuels scolaires ne laissent que peu de places aux hérö̈nes (en dehors de Jeanne d'ARC, elles sont relativement absentes) et/ou aux auteures et créatrices, alors que les autres médias (télévision, radio, presse, Internet) et supports éducatifs (jeux, livres, BD, dessins animés éducatifs) leur offrent une place croissante : selon Maya GöTZ $32 \%$ des personnages des émissions de télévision pour enfants sont de sexe féminin (2008). 
Si la plupart des utilisateurs et utilisatrices (élèves ou enseignant-e-s) des manuels pensent que les rapports hommes/femmes sont pris en considération dans les manuels, ils s'accordent pour souligner la persistance de stéréotypes sexistes. En fait, ces derniers apparaissent quand on y prête attention : ce sont les effets de la socialisation genrée que d'occulter les rapports sociaux de sexe. Cet impensé est dû en partie à un processus d'essentialisation de la construction du genre (des filles qui jouent à la poupée : «c'est normal!»), qui nie ou omet précisément la dimension construite de ces rapports de sexe et ils cachent ainsi une partie de la réalité discriminatoire et stéréotypée des manuels. À la question "pouvez-vous décrire un exemple de stéréotype à l'égard des femmes que vous avez rencontré dans l'un des manuels que vous utilisez ou avez utilisé ?», les réponses peuvent être classées en quatre catégories : les femmes " femmes de ménage » ${ }^{16}$ qui arrivent en tête suivies des femmes actives " aux petits métiers dévalorisés ${ }^{17}$, des femmes « fragiles », « soumises » ou victimes ${ }^{18}$ et enfin des " gentilles idiotes » ou femmes " objet du désir masculin », notamment dans l'analyse des publicités ${ }^{19}$. Quant aux élèves, certains font des constats significatifs, comme cet élève de $6 \mathrm{e}:$ «dans le livre de maths, c'est les gars qui ont raison! ", jamais une fille n'a la bonne réponse. Si la méthodologie de l'étude n'a pas permis de mesurer l'impact des contenus sur les élèves, cet exemple montre que ceux-ci y sont sensibles et peuvent être en mesure de les lire avec un œil critique.

31 Compte tenu de l'importance du langage dans notre capacité à nous projeter et nous identifier (YAGUELLO, 1978, 1989), il importe que les manuels scolaires reflètent un monde pensé, décrit et écrit au féminin comme au masculin et qu'un travail de déconstruction des stéréotypes soit généralisé.

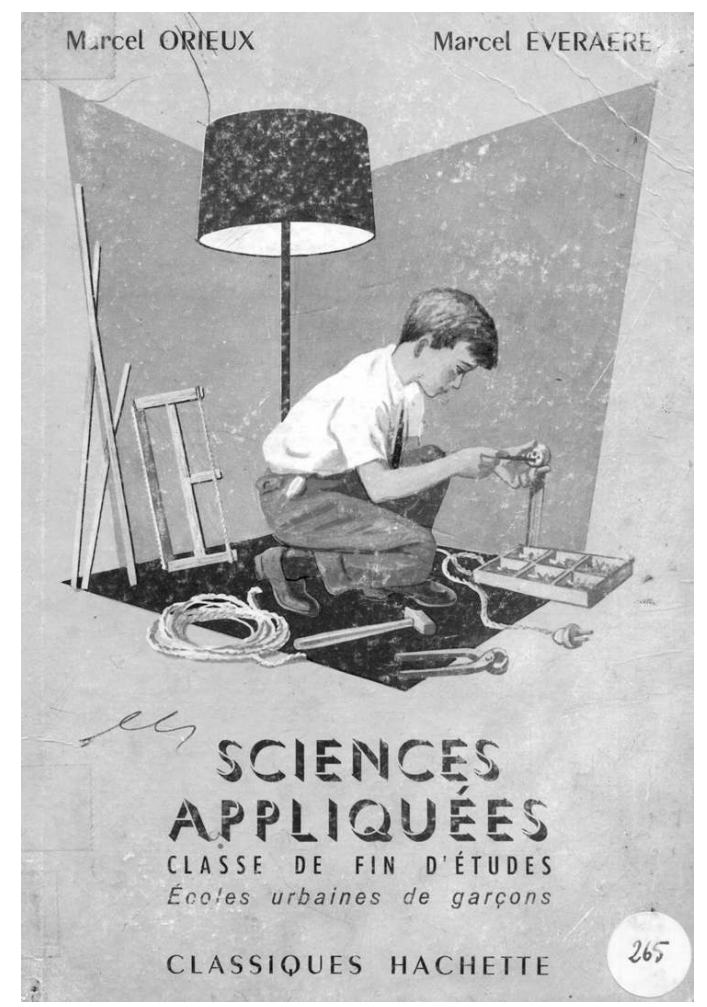

Sciences appliquées, classe de fin d'études, écoles urbaines de garçons, Marcel ORIEUX et Marcel EVERAERE, Paris : HACHETTE, 1958, 190 p. - Couverture - Source : CEDRHE. 


\section{BIBLIOGRAPHIE}

Associations femmes et sciences, Femmes et mathématiques et Femmes ingénieurs, Les femmes et les sciences. Au-delà des idées reçues, 2006.

BAERLOCHER E., Barbie contre Action Man! Le jouet comme objet de socialisation dans la transmission des rôles stéréotypiques de genre, in DAFFLON-NOVELLE A. (dir.), Filles-garçons : socialisation différenciée ? Grenoble : Presses universitaires de Grenoble, 2006 BAtTAGliola F., Histoire du travail des femmes. Paris : La Découverte, Coll. Repères, 2004, 2000 BAUDELOT C., ESTABLET R., Allez les filles ! Paris : Éditions du Seuil, 1992 BAUdeLOT C., ESTABLET R., Quoi de neuf chez les filles ? Entre stéréotypes et libertés. Paris : Nathan, 2007

BERTON-SCHMITT A., La place des femmes dans les manuels d'histoire du secondaire, in L'Observatoire de la parité. Entre les hommes et les femmes. Rapport IEP Grenoble. Université Pierre Mendès-France, 2005

CROMER S., BRUGEILLES C., Analyser les représentations du masculin et du féminin dans les manuels scolaires. Paris : Ceped, Collection « Les clefs pour... », 2005

CROMER S., TURIN A., Quels modèles pour les filles? Une recherche sur les albums illustrés. Paris : Association Du Côté des Filles, 1997

CROMER S., TURIN A., Que voient les enfants dans les livres d'image? Des réponses sur les stéréotypes. Paris : Association Du Côté des Filles, 1998

DECROUX-MASSON A., Papa lit, maman coud. Les manuels scolaires en bleu et rose. Paris : Denoël/ Gonthier, 1979

DETREZ C., Il était une fois le corps... La construction biologique du corps dans les encyclopédies pour enfants, Sociétés contemporaines, n59-60, 2006, p 161-177

DJIDER Z., MURAT F., Robin I., Motivation et performances scolaires : les filles creusent l'écart, Insee

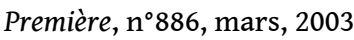

DUBy G., PERROT M., Histoire des femmes en Occident. Paris : Perrin, Coll. Tempus, 2002

FERRAND M., Féminin, masculin. Paris : La découverte, Coll. Repères, 2004, 2001

FRAISSE G., Les femmes et leur histoire. Paris : Gallimard, Coll. Folio histoire, 1998

GÖTZ M., Children's television worldwide : Gender Representation (2007-2008), 2008

LAUFER J., MARRY C., MARUANI M. (dir.), Le travail du genre. Paris : La découverte/MAGE, 2003

MARUANI, M., (dir.) Les nouvelles frontières de l'inégalité. Hommes et femmes sur le marché du travail. Paris : La découverte, Coll. Recherches, 1998

MARUANI, M., Travail et emploi des femmes. Paris : La découverte, Coll. Repères, 2003, 2000

PERROT M., Les Femmes ou les silences de l'histoire. Paris : Flammarion, 1998 
RIGNAULT S., RICHERT P., La représentation des hommes et des femmes dans les livres scolaires. Paris : La documentation française, 1997

SINGLY, F., de, Le soi, le couple, la famille. Paris : Nathan, 1996

TERRAIL J.-P., La supériorité scolaire des filles, chapitre II, La scolarisation de la France. Critique de l'état des lieux. Paris : La Dispute, 1997

TISSERANT P., WAGNER A.-L. (sous la dir.), Place des stéréotypes et discriminations dans les manuels scolaires, Rapport pour la HALDE, 2008

Yaguello, M., (1978), Les mots et les femmes. Paris : Payot et Rivages, 2002

yaguello, M., Le sexe des mots. Paris : Belfond, Coll. Point Virgule, 1989

\section{Corpus de manuels}

\section{Éducation civique (juridique et sociale)}

Éducation civique 6e, Grandir ensemble, livre de l'élève. Paris : HATIER, 2005

Éducation civique 5e, Grandir ensemble, livre de l'élève. Paris : HATIER, 2006

Éducation civique 4e, Demain, citoyens, livre de l'élève. Paris : NATHAN, 2006

Éducation civique 3e, Livre de l'élève. Paris : HACHETTE éducation, 2007

Éducation civique, juridique et sociale 2de. Fichier de l'élève. Paris : BORDAS, 1999

Éducation civique, juridique et sociale 1re. Paris : MAGNARD, 2001

Éducation civique, juridique et sociale Terminale. Paris : MAGNARD, 2002

\section{Histoire- géographie}

Histoire-Géographie 5e. Paris : HATIER, 2005

Histoire-Géographie 4e. Paris : HATIER, 2006

Histoire-Géographie Terminale STG. Paris : MAGNARD, 2007

Histoire-Géographie bac pro. Paris : NATHAN, 2007

Histoire terminale L-ES-S. Paris : HACHETTE éducation, 2004

\section{Français}

Textes et compagnie 6e. Paris : NATHAN, 2005

Lettres vives 4e. Paris : HACHETTE éducation, 1998

Terres littéraires 2de. Paris : HATIER, 2006

\section{Anglais}

New Spring 6e. Paris : HACHETTE éducation, 2007

New Step in 4e. Paris : HATIER, 2002 
Insight 2de. Paris : HATIER, 2005

New goals plus BEP. Paris : FOUCHER

\section{Mathématiques}

Triangle 3e, livre de l'élève. Paris : HATIER, 2003

Mathématiques 5e, Collection Phare, Paris : HACHETTE Éducation, 2006

Sigma, 1re STG. Paris : FOUCHER, 2005

\section{Sciences de la vie et de la terre}

SVT 6e. Paris : BELIN, 2005

SVT 5e. Paris : BELIN, 2006

SVT 4e. Paris : NATHAN, 2007

SVT 3e. Paris : BORDAS, 1999

SVT 1re S. Paris : BORDAS, 2007

SVT Terminale. Paris : BORDAS, 2002

\section{Sciences Economiques et Sociales}

Sciences économiques et sociales Terminale. Paris : HATIER, 2007

\section{NOTES}

1. TISSERAnt P., WAgner A.-L. (sous la dir.), Place des stéréotypes et discriminations dans les manuels scolaires, Rapport pour la HALDE, mars 2008.

2. Cette haute autorité, créée par la loi du 30 décembre 2004, a pour mission officielle, d'une part, de lutter contre les discriminations (aide à l'identification des pratiques discriminatoires, conseil juridique, aide à l'établissement de la preuve, instruction des dossiers) et d'autre part, de promouvoir l'égalité (émission d'avis et de recommandations auprès du gouvernement, du Parlement et des autorités publiques pour lutter contre les discriminations, afin d'améliorer les textes de loi, de faire progresser l'état du droit français dans ce domaine). Depuis le 29 mars 2011, la HALDE fait partie de l'institution 'défenseur des droits (voir http://defenseurdesdroits.fr/ sinformer-sur-le-defenseur-des-droits/linstitution).

3.

http://media.education.gouv.fr/file/special_6/51/8/

Programme_hist_geo_education_civique_5eme_33518.pdf

4. Faute de mieux, nous avons choisi d'utiliser les termes « origine culturelle réelle ou supposée » et «minorités visibles » pour interroger les discriminations dites ethniques et culturelles afin de circonscrire l'analyse aux situations les plus discriminatoires.

5. La récente polémique (janvier-février 2010) autour du film d'animation réalisé par Sébastien WATEL, Le baiser de la lune, visant à travailler avec les élèves de CM1-CM2 la question de l'homophobie met en évidence les nombreux freins pour une sensibilisation à la diversité sexuelle et au respect de celle-ci. 
6. Les statistiques récentes montrent combien ces situations et occupations sociales restent des réalités dominantes. Pour autant, il apparaît réducteur et stéréotypé d'évacuer ou nier un certain nombre de transformations sociales plaçant les femmes dans des positions sociales diversifiées dans les sociétés contemporaines.

7. Parfois même à des postes de responsabilité, et dans quasiment toutes les sphères sociales. Certes avec les nombreuses difficultés et inégalités, notamment en termes de salaires ou d'accès à certains espaces encore pré carré de la gent masculine (MARUANI, 1998 ; LAUFER, MARRY, MARUANI, 2003 ; FERRAND, 2004).

8. Soulignons que la dernière édition (2010) du manuel pour les élèves de 5e a été révisée. Un chapitre, "La réduction des inégalités ", aborde la thématique de l'égalité entre les filles et les garçons, entre les femmes et les hommes. Un corpus de documents présente les principales étapes de la conquête de l'égalité tout en notant que même si « la situation s'est améliorée, il reste des progrès à réaliser ». Les élèves sont amenés entre autres à réfléchir sur la situation des femmes des années 1800 et à celle des femmes d'aujourd'hui, aux difficultés qu'elles ont pu rencontrer tout au long de cette marche vers l'égalité, et à l'égalité dans l'éducation. Olympe de Gouges, les suffragettes et le Mouvement de libération des femmes sont présentés dans un focus sur « les femmes luttent pour l'égalité ». Certaines avancées législatives sont exposées : « 1965 : la femme peut ouvrir un compte bancaire » « 2000: loi sur la parité en politique », « 2006: autorisation pour les femmes, comme l'homme, de donner son nom de famille à ses enfants ». Un document est également consacré au prix de vocation scientifique et technique des filles (PVST), qui vise à soutenir les jeunes filles qui font le choix de s'engager dans des filières où elles sont peu nombreuses. Les notions de stéréotype et d'images stéréotypées sur les rôles sociaux des femmes et des hommes reçoivent une attention particulière. Les élèves ont par ailleurs la possibilité en fin d'activité de créer une affiche montrant qu'il n'y a pas de métiers exclusivement réservés aux femmes et aux hommes.

9. Cet encadré s'accompagne d'une photographie avec quatre jeunes bénévoles, dont un homme, au premier plan, qui pousse une brouette lourdement chargée de pierres, et trois femmes, à l'arrière-plan, qui ramassent des pierres, tamisent de petites quantités de sable et remplissent d'autres brouettes. C'est une division du travail somme toute des plus classiques mise en scène.

10. Qui plus est, la part des hommes dans la profession est de $80 \%$.

11. Première femme (1850-1891) à avoir obtenu une thèse en mathématiques et à devenir professeure d'université, à voir ses travaux primés par l'Académie des sciences de Paris.

12. Auteure d'une traduction et de commentaires des travaux de NEWTON qui font autorité et classée parmi les dix plus grands savants de son époque (1706-1749).

13. Si ces femmes ne disent sans doute pas grand-chose à la plupart d'entre nous (et pour cause, rencontrer leurs noms et travaux suppose de faire des études spécialisées poussées), elles sont néanmoins d'éminentes chercheures, reconnues dans leur discipline.

14. Avec un texte de COLETTE, La maison de Claudine (1922), de Simone DE BEAUVOIR, Mémoires d'une jeune fille rangée (1958) et de Mariama BÂ, Une si longue lettre (1979).

15. Avec les textes de Olympe DE GOUGE, Déclaration des droits de la Femme et de la Citoyenne (1791), de Simone DE BEAUVOIR, Le deuxième sexe (1949), de Marguerite YOURCENAR, Les yeux ouverts (1980), de Giselle HALIMI, La cause des femmes (1974) et de Élisabeth BADINTER, Fausse route (2003).

16. "C'est toujours la femme qui s'occupe des enfants, fait la cuisine.... "; « La femme s'occupe des enfants et des tâches ménagères dans les illustrations et les dialogues proposés "; "Le shopping et la cuisine sont des activités typiquement féminines ».

17. "Très rare de voir des femmes chefs d'entreprise "; « La femme ne peut exercer que des fonctions d'exécution»"; "Au chapitre des professions elles ne sont jamais ingénieurs ou architectes etc. ». 
18. «Très souvent, quand un sportif est représenté (dessin ou plus souvent photographie), c'est un homme. Les femmes seraient donc moins sportives que les hommes, et comme ce sont souvent des professionnels ou des « champions » connus, cela pourrait laisser penser que les femmes sont moins capables de bonnes performances... »; «L'histoire d'une jeune adulte qui veut quitter la maison, mais qui se querelle avec ses parents (souvent le père) car il ne veut pas la laisser partir. Elle a souvent un frère, qui lui a eu la permission de faire ce qu'il voulait... ».

19. «Dans les manuels de technologie, les représentations masculines sont plutôt à leur avantage alors que les représentations féminines sont tournées en gentille dérision (dubitatives devant un problème ou une machine outil compliquée ...)».

\section{RÉSUMÉS}

L'étude repose sur l'analyse de 29 manuels scolaires français du point de vue des représentations des rapports de genre véhiculées et de 60 entretiens avec des enseignant-e-s, des élèves et des éditeurs-éditrices. Après une présentation rapide des principaux résultats du rapport à l'origine de cette contribution, qui incluait aussi les représentations des origines socioculturelles, des générations, des sexualités et des handicaps, nous montrerons comment les rapports de genre restent stéréotypés et fonctionnent sur des routines langagières et iconographiques qui ne favorisent pas la diversité des modèles d'identification des sexes.

The study is based on the analysis of 29 French textbooks about representations of gender rapport and 60 interviews with teachers, pupils and publishers. After the principals results of the rapport at the origin of this contribution, which included also sociocultural and generational factors, the sexuality and the handicap, we shall show how gender's always stereotypical and works as linguistic and iconographic routines which do not facilitate the variety of the models of identification of the sex.

\section{INDEX}

Keywords : diversity, gender, stereotype, textbook

Mots-clés : diversité, genre, manuel scolaire, stéréotypes

\section{AUTEUR}

SABRINA SINIGALIA-AMADIO

2L2S/ERASE ; Université Paul-Verlaine, France 